\title{
MICROCAVITIES
}

\section{Pyramidal resonators}
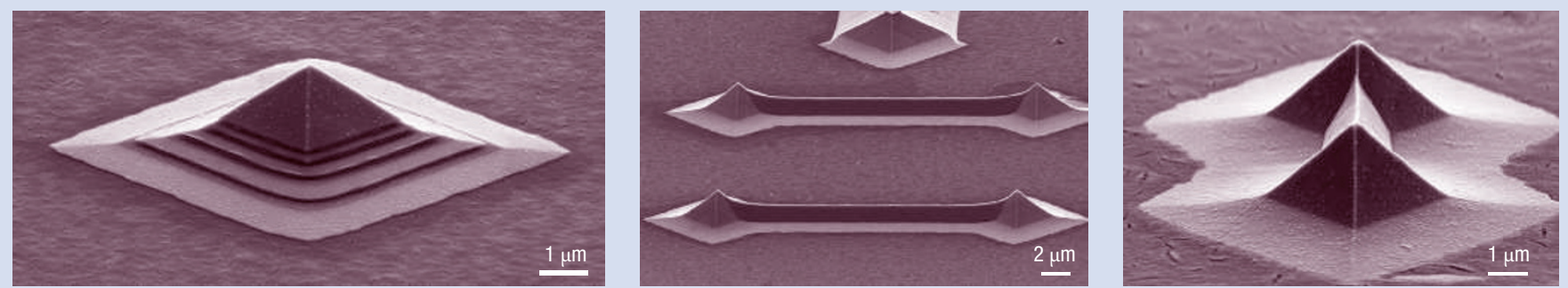

Microscale three-dimensional pyramid-shaped GaAs structures embedded with InGaAs quantum-dot light emitters have been developed by Frank Weber and co-workers at the University of Karlsruhe, Germany. The team says that once optimized, the microcavity resonators have the potential for small mode volumes and high $Q$ factors, and could be used as fibre-coupled single-photon sources (Appl. Phys. Lett. 90, 161104; 2007).

The resonators rely on internal reflection of light from the tilted pyramid facets to achieve strong confinement of light in all three spatial dimensions with low loss. The researchers fabricated the structures using a combination of molecular-beam epitaxy, electron-beam lithography and wet chemical etching. They report that the techniques allow versatile control of cavity shape, size and facet angle, as well as fabrication of more complex structures.

The researchers grew GaAs with a refractive index of 3.5 on top of a highly reflective GaAs/AlAs distributed Bragg reflector, which acts as a base plane for the pyramidal resonator. The pyramidal cavities also contain three InGaAs quantum-dot layers placed a few hundred nanometres above the base plane. When the quantum dots are excited with continuous-wave laser at $532 \mathrm{~nm}$, the cavities serve as an internal light source, emitting in the range from $900 \mathrm{~nm}$ to $1,000 \mathrm{~nm}$. In microphotoluminescence measurements carried out at increasing temperatures for pyramidal resonators with base lengths of $1.9 \mu \mathrm{m}$ and $2.1 \mu \mathrm{m}$ and heights of $780 \mathrm{~nm}$ and $850 \mathrm{~nm}$, respectively, the team observed spectra consisting of many peaks, some of which corresponded to optical resonant modes in the cavity.

Weber and colleagues have also fabricated a more complex, tent-like structure containing just a few quantum dots, and observed localized emission through the structure's facets. They envisage that the tent-like structure might be of use in realizing singlephoton emitters, where a photon emitted by a quantum dot can be efficiently collected by the cavity and coupled to an optical fibre for subsequent use. Although the reported $Q$ factor is not impressively high, the researchers expect it to be improved by optimizing the facet reflectivity of the pyramidal cavities.

Rachel Won

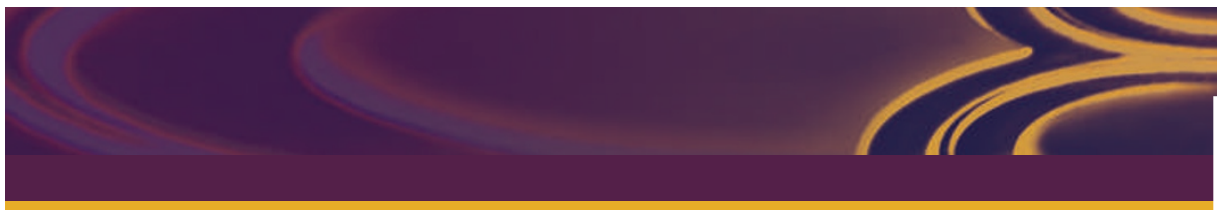

\section{News \& Views contributions}

The News \& Views section is where new advances in photonics, reported in published papers or at meetings, are communicated to a broad audience. Many News \& Views pieces are linked to Articles and Letters that appear in Nature Photonics, but they can also focus on important papers that are published elsewhere. Unsolicited contributions will not normally be considered, although we welcome advance warning about forthcoming papers of exceptional significance. As a general guideline,

News \& Views pieces are about 800-900 words long, with one or two display items (figures, boxes or tables). They should make clear the advance (the "news") and communicate a sense of excitement, yet provide a critical evaluation of the work within the context of the rest of the field. We encourage personal views, criticisms and predictions, but authors should not refer to their own work, except in passing.

Detailed guidelines are available upon request from naturephoton@nature.com and on the website for Nature Photonics (www.nature.com/naturephotonics). nature photonies Optical memory comes of age

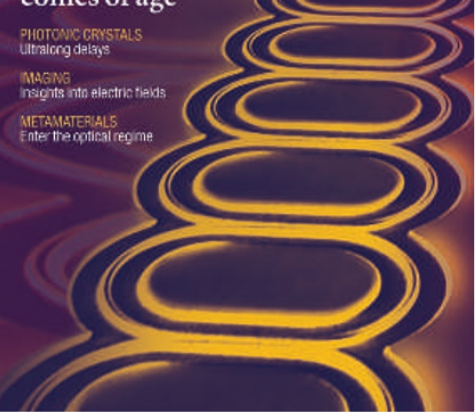

